\title{
Generalized version of chiral Schwinger model in terms of chiral bosonization
}

\author{
Safia Yasmin ${ }^{\mathrm{a}}$ \\ Indas Mahavidyalaya, Indas, Bankura, West Bengal 722205, India
}

Received: 10 July 2020 / Accepted: 18 February 2021 / Published online: 1 March 2021

(C) The Author(s) 2021

\begin{abstract}
The $(1+1)$ dimensional generalized model where vector and axial vector interaction get mixed up with different strength is considered. Imposing a chiral constraint, the model can be expressed in terms of chiral boson. Then the theoretical spectra of this model has been determined in both the Lagrangian and Hamiltonian formalism. It is found that the massless degrees of freedom disappears from the spectra and the photon acquires mass as well. Imposition of chiral constraint brings a disaster so far as Lorentz invariance is concerned. An attempt has been made here to show the physical Lorentz invariance explicitly using Poincaré algebra.
\end{abstract}

\section{Introduction}

Free chiral bosons in two dimensions recently become an important phenomena mainly in the context of string theories [1-8]. The chiral boson is coupled with the gauge field has given interacting field theoretical models. From long ago much attention has been paid to qualitative study of chiral boson and gauging of it in $(1+1)$-dimensional quantum field theory [1-6]. Siegel proposed a manifestly covariant lagrangian formulation for chiral bosons [2] in two dimensions. However, it turns out that the reparametrization symmetry was not obeyed at the quantum level due to the presence of gravitational anomaly [6,7]. Floreanini and Jackiw described an alternative lagrangian but there manifestly Lorentz covariance was absent [4]. Bellucci, Golterman, and Petcher [5] considered the interaction of chiral bosons with Abelian and non-Abelian gauge fields and discussed the chiral schwinger model as an example. Labastida and Ramallo had taken chiral bosons coupled to Abelian gauge fields using Becchi-Rouet-Stora-Tyutin (BRST) formalism [6]. After that Harada reexamined the chiral schwinger model by using chiral bosonization [1]. The author has obtained lagrangian of Floreanini-Jackiw type chiral boson from the

a e-mail: safiayasmin01513@gmail.com (corresponding author) chiral schwinger model by imposing a sort of chiral constraint in its phase space.

In this paper, we express the conventional bosonic generalised version of schwinger model (GVSM) in terms of chiral boson by imposing the chiral constraint $\pi_{\phi}-\phi^{\prime}$ in its phase space $[1,9]$. This lagrangian consists of fewer degrees of freedom than the conventional bosonic one. Furthermore, we quantize the said model with the help of Lagrangian and Hamiltonian formulation and find out the spectra. Therefore correlation between these two are made. Imposition of chiral constraint to any arbitrary model has spoiled the manifestly Lorentz covariance. We have shown that despite the manifestly Lorentz covariance of this theory the physical Lorentz invariance is maintained in the reduced phase space.

In this context, we consider a model where both vector and axial vector interaction are accomplished with different strength [10-14]. The model which is under consideration may be regarded as a generalised version of QED (GVQED). All the fundamentally distinct interaction and their mixing are defined by the model [10-13]. Bassetto et al. [10] suggested the generalised version of schwinger model (GVSM), i.e., a vector and axial-vector theory characterized by $a$ parameter which interpolates between pure and chiral schwinger models. One can achieve both the schwinger model and the chiral schwinger model [4] through the different choices of its mixing weight factor of interaction. For $r=0$ one recovers the vector schwinger model $[15,16]$; for $r= \pm 1$ one can gets the chiral schwinger model. The model is specified by two parametrs $r$ and $a$ where $\mathrm{r}$ measures the relative strength of right and left couplings while $a$ parametrizes the anomaly ambiguity.

Schwinger [17] solved the two-dimensional electrodynamics with massless spinors, and described some interesting features, for example, dynamical generation of a mass for the gauge field, fermion confinement, and so on. Hagen [18] initially studied the chiral formalism of the schwinger model, known as the chiral schwinger model (CSM). After 
that Jackiw and Rajaraman [4] investigated it in detail. They have revealed that the CSM, which consists of a U(1) gauge field coupled to chiral fermions in two-dimensional spacetime. In addition, a generalized version of schwinger model (GVSM) $[19,20]$, i.e., a generalized theory where the left and right fermions couple to the gauge field with different weights was taken in order to shed light on some physical aspects of the CSM [21,22]. Generalized version of schwinger model (GVSM) [14] is of considerable interest because of its ability to describe different physical aspects. It will therefore be interesting if we express the GVSM in terms of chiral boson. So study of this version would certainly be interesting.

This paper is organized as follows. In Sect. 2, we impose a chiral constraint in the phase space of the generalised schwinger model and obtain bosonic generalised version of schwinger model (GVSM) in terms of chiral boson. In Sect. 3, physical spectrum of the model (GVSM in terms of chiral boson) is determined in the Lagrangian formulation. Section 4 is devoted to determine the physical spectrum of the same model through the Hamiltonian formulation and also try to find out correlation with the spectrum obtained in Sect. 3 . In Sect. 5, we carry out the Poincaré algebra to verify physical Lorentz invariance of the model. End section consists of conclusion.

\section{Imposition of chiral constraint}

A model where we find both vector and axial vector theory characterized by $a$ parameter is given by the following generating functional $[10,11,13]$

$Z(A)=\int d \psi d \bar{\psi} e^{\left[i \int d^{2} x L_{F}\right]}$,

where

$L_{F}=\bar{\psi} \gamma^{\mu}\left[i \partial_{\mu}+e \sqrt{\pi} A_{\mu}\left(1-r \gamma_{5}\right)\right] \psi$.

One can regularize the model to remove singularity, this singularity arise from the integration over the fermionic degrees of freedom $[10,11,13]$. Now writing down the generating functional in terms of auxiliary scalar field $\phi$, which turns out to the following

$Z(A)=\int d \phi e^{\left[i \int d^{2} x \mathcal{L}_{\mathcal{B}}\right]}$,

with [23]

$$
\begin{aligned}
\mathcal{L}_{\mathcal{B}}= & \frac{1}{2}\left(\dot{\phi}^{2}-\phi^{\prime 2}\right)+e\left(A_{0} \phi^{\prime}-A_{1} \dot{\phi}\right)+\operatorname{er}\left(A_{0} \dot{\phi}-A_{1} \phi^{\prime}\right) \\
& +\frac{e^{2}}{2} a\left(A_{0}^{2}-A_{1}^{2}\right)
\end{aligned}
$$

Here $a$ represents a real parameter which expresses the ambiguity in the bosonization procedure $[10,13]$.

The momentum corresponding to the field $\phi$ is found out to be

$\frac{\partial \mathcal{L}_{\mathcal{B}}}{\partial \dot{\phi}}=\pi_{\phi}=\dot{\phi}-e A_{1}+\operatorname{er} A_{0}$.

The canonical Hamiltonian is $H_{c}=\pi_{\phi} \dot{\phi}-L$.

We obtain the following canonical Hamiltonian with the help of Legendre transformation:

$$
\begin{aligned}
H_{B}= & \int d x\left[\frac{1}{2}\left(\pi_{\phi}^{2}+\phi^{2}\right)+e \pi_{\phi}\left(A_{1}-r A_{0}\right)\right. \\
& \left.-\frac{e^{2}}{2} a\left(A_{0}^{2}-A_{1}^{2}\right)+\frac{1}{2} e^{2}\left(A_{1}-r A_{0}\right)^{2}+e \phi^{\prime}\left(r A_{1}-A_{0}\right)\right] .
\end{aligned}
$$

We impose here the following chiral constraint,

$\omega(x)=\pi_{\phi}(x)-\phi^{\prime}(x) \approx 0$.

It turns out that the chiral constraint $\omega(x)$ is second class:

$[\omega(x), \omega(y])]=-2 \delta^{\prime}(x-y)$.

After imposing the constraint $\omega \approx 0$, and integrating out $\pi_{\phi}$ of the field $\phi$, we have obtained the generating functional in the following form:

$$
\begin{aligned}
Z_{C H} & =\int d \phi d \pi_{\phi} \delta\left(\pi-\phi^{\prime}\right) \sqrt{\operatorname{det}[\omega, \omega]} e^{i \int d^{2} x\left[\pi_{\phi} \dot{\phi}-\mathcal{H}_{B}\right]} \\
& =\int d \phi e^{i \int d^{2} x \mathcal{L}_{C H}} .
\end{aligned}
$$

with

$$
\begin{aligned}
\mathcal{L}_{C H}= & \dot{\phi} \phi^{\prime}-\phi^{\prime 2}+e\left(A_{0} \phi^{\prime}-A_{1} \phi^{\prime}\right)+\operatorname{er}\left(A_{0} \phi^{\prime}-A_{1} \phi^{\prime}\right) \\
& +\frac{e^{2}}{2} a\left(A_{0}^{2}-A_{1}^{2}\right)-\frac{1}{2} e^{2}\left(A_{1}-r A_{0}\right)^{2} .
\end{aligned}
$$

This Eq. (10) is the result of systematic description of the previous Lagrangian (4) in terms of chiral boson. Other wise we say that we have obtained the gauged lagrangian for chiral boson (10) from the bosonized lagrangian of generalised version of schwinger model (GVSM) just by imposing the chiral constraint in its phase space. The first two terms are described the kinetic terms of the chiral boson [4-6]. It is also a matter of investigation whether this model remains solvable after imposition of this constraint. Therefore we proceed to quantize the model described by the lagrangian (10) with the help of Lagrangian and Hamiltonian formalism.

\section{Theoretical spectrum in the Lagrangian formulation}

We quantize the theory with the help of Lagrangian formulation to determine the spectrum. The starting lagrangian den- 
sity is

$$
\begin{aligned}
\mathcal{L}_{C H}= & \dot{\phi} \phi^{\prime}-\phi^{\prime 2}+e\left(A_{0} \phi^{\prime}-A_{1} \phi^{\prime}\right)+e r\left(A_{0} \phi^{\prime}-A_{1} \phi^{\prime}\right) \\
& +\frac{e^{2}}{2} a\left(A_{0}^{2}-A_{1}^{2}\right)-\frac{1}{2} e^{2}\left(A_{1}-r A_{0}\right)^{2}+\frac{1}{4} F_{\mu \nu} F^{\mu \nu} .
\end{aligned}
$$

The equations of motion for the field $\phi, A_{0}, A_{1}$, are

$$
\begin{aligned}
& 2\left(\dot{\phi}^{\prime}-\phi^{\prime \prime}\right)+e\left(A_{0}^{\prime}-A_{1}^{\prime}\right)+e r\left(A_{0}^{\prime}-A_{1}^{\prime}\right)=0, \\
& A_{0}^{\prime \prime}-\dot{A}_{1}^{\prime}-a e^{2} A_{0}+e^{2} r^{2} A_{0}-e^{2} r A_{1}-e \phi^{\prime}-e r \phi^{\prime}=0, \\
& \ddot{A_{1}}-\dot{A}_{0}{ }^{\prime}+a e^{2} A_{1}+e \phi^{\prime}+e r \phi^{\prime}+e^{2} A_{1}-e^{2} r A_{0}=0 .
\end{aligned}
$$

To solve the Eqs. (12), (13), (14) we introduce the ansatz for the fields $\phi$ and $A_{\mu}$ are

$$
\begin{aligned}
\phi & =-\frac{1}{e\left(1+a-r^{2}\right)} F, \\
A_{\mu} & =-\frac{1}{e^{2} a}\left[\frac{\left(a-r^{2}\right)}{\left(1+a-r^{2}\right)} \tilde{\partial_{\mu}} F+\frac{r}{\left(1+a-r^{2}\right)} \partial^{\mu} F\right] .
\end{aligned}
$$

Equation (12) gives

$2\left(\dot{\phi}-\phi^{\prime}\right)=-e(r+1)\left(A_{0}-A_{1}\right)$,

and the Eq. (16) provides

$\dot{A_{1}}-A_{0}^{\prime}=-\frac{1}{e^{2} a}\left[\frac{\left(a-r^{2}\right)}{\left(1+a-r^{2}\right)}\right] \square F$.

To solve the Eqs. (13) and (14), we put the ansatz for the fields $A_{\mu}$ and $\phi$ and also used the relation of (17) and (18). Finally we get the following solution of $F$

$$
\left(\square+m^{2}\right) F=0 .
$$

$m^{2}$ is defined as

$m^{2}=\frac{e^{2} a\left(1+a-r^{2}\right)}{\left(a-r^{2}\right)}$.

We have found that the spectrum consists of a massive boson with mass term $m^{2}=\frac{e^{2} a\left(1+a-r^{2}\right)}{\left(a-r^{2}\right)}$ [23]. Massive boson as usual can be considered as photon acquire mass via dynamical symmetry breaking. The massless degrees of freedom does not appear in this situation. Massless degrees of freedom can be considered as fermion. So fermion gets confined here. We also mention that the squared of mass term consists of an additional factor $\frac{1}{\pi}$ in article [10]. It signifies that the mass term is canonically normalized. $\frac{e}{\sqrt{\pi}}$ represents the mass of a free field. If we use $\frac{e}{\sqrt{\pi}}$ in place of $-e$ then we recover the result appearing in [10]. The details discussion is deffered to Appendix.

\section{Constraint analysis and determination of the theoretical spectrum through Hamiltonian formulation}

An attempt is also made to extract out the real physical canonical pairs embedded within the phase space of the theory described by the lagrangian (11) through the Hamiltonian formalism $[1,13]$. At first it is needed to calculate the momenta of the fields describing the theory.

The momentum corresponding to the fields $\phi, A_{0}, A_{1}$ are

$$
\begin{aligned}
\pi_{\phi} & =\phi^{\prime}, \\
\pi_{0} & =0, \\
\pi_{1} & =\dot{A_{1}}-A_{0}^{\prime} .
\end{aligned}
$$

Here $\omega_{1}=\pi_{0} \approx \omega_{2}=\pi_{\phi}-\phi^{\prime} \approx$ identifies as the primary constraints of the theory $[24,25]$. From Legendre transformation, we have obtained the canonical Hamiltonian of the system

$$
\begin{aligned}
H_{c}= & \phi^{\prime 2}+\frac{\pi_{1}^{2}}{2}+\pi_{1} A_{0}^{\prime}-\frac{1}{2} e^{2}\left(A_{1}-r A_{0}\right)^{2} \\
& +e \phi^{\prime}\left(A_{1}-r A_{0}\right)-e\left(A_{0} \phi^{\prime}-r A_{1} \phi^{\prime}\right) \\
& -\frac{a e^{2}}{2}\left(A_{0}^{2}-A_{1}^{2}\right) .
\end{aligned}
$$

Time consistency of primary constraint with respect to the Hamiltonian gives secondary constraint,

$\omega_{3}=\pi_{1}^{\prime}+e^{2}\left(a-r^{2}\right) A_{0}+e^{2} r A_{1}+e r \phi^{\prime}+e \phi^{\prime}$.

The constraints are all weak conditions at this stage. To insert it as a strong condition, we need to calculate the value of $A_{0}$ from the Eq. (25). From Eq. (25), we get the following

$A_{0}=-\frac{1}{e^{2}\left(a-r^{2}\right)}\left(\pi_{1}^{\prime}+e r \phi^{\prime}+e \phi^{\prime}+e^{2} r A_{1}\right)$.

Putting the expression of $A_{0}$ in Eq. (24), we can get the reduced Hamiltonian

$$
\begin{aligned}
H_{R}= & \frac{\pi_{1}^{2}}{2}+\frac{\pi_{1}^{\prime 2}}{2 e^{2}\left(a-r^{2}\right)}+\frac{a \phi^{\prime 2}}{\left(2\left(a-r^{2}\right)\right.}+\frac{e^{2}}{2} \frac{a\left(1+a-r^{2}\right)}{\left(a-r^{2}\right)} A_{1}^{2} \\
& +\frac{\left(1+a-r^{2}\right)}{\left(a-r^{2}\right)} \frac{\phi^{\prime 2}}{2}+\frac{r \phi^{\prime} \phi^{\prime}}{\left(a-r^{2}\right)}+\frac{\phi^{\prime} \pi_{1}^{\prime}}{e\left(a-r^{2}\right)}+\frac{r \phi^{\prime} \pi_{1}^{\prime}}{e\left(a-r^{2}\right)} \\
& +e r \frac{\left(1+a-r^{2}\right)}{\left(a-r^{2}\right)} A_{1} \phi^{\prime}+e \frac{a}{\left(a-r^{2}\right)} A_{1} \phi^{\prime}+\frac{r \pi_{1}^{\prime} A_{1}}{\left(a-r^{2}\right)} .
\end{aligned}
$$


The reduced Hamiltonian can be expressed in the following form

$$
\begin{aligned}
H_{R}=\int & d x\left[\phi^{\prime 2}+\frac{\pi_{1}^{2}}{2}+\frac{e^{2}}{2}(1+a) A_{1}^{2}-e \phi^{\prime} A_{1}(r+1)\right. \\
& \left.+\frac{\eta^{2}}{2 e^{2}\left(a-r^{2}\right)}\right],
\end{aligned}
$$

where

$\eta=\pi_{1}^{\prime}+e r \phi^{\prime}+e \phi^{\prime}+e^{2} r A_{1}$.

Poisson brackets get invalidated for this reduced Hamiltonian. This reduced Hamiltonian however remains consistent with the Dirac brackets. So it is essential to calculate the Dirac bracket between the fields describing the theory. The Dirac bracket between the two variables A and B is defined as

$$
[A, B]^{*}=[A, B]-\int\left[A(x), \omega_{i}(\eta)\right] C_{i j}^{-1}\left[\omega_{i}(\eta), B(x)\right],
$$

here $C_{i j}^{-1}$ is given by

$$
\int C_{i j}^{-1}(x, z)[\omega(z), \omega(y)] d z=1 .
$$

This theory consists of three constraints. These constraints $\omega_{i}^{\prime} s$ are contruct the second class set. The matrix $C_{i j}^{-1}$ is

$C_{i j}^{-1}=\left(\begin{array}{ccc}0 & \frac{1}{e^{2}\left(a-r^{2}\right)} & \frac{(1+r)}{2 e\left(a-r^{2}\right)} \\ \frac{-1}{e^{2}\left(a-r^{2}\right)} & 0 & 0 \\ -\frac{(1+r)}{2 e\left(a-r^{2}\right)} & 0 & \frac{1}{2 \delta}\end{array}\right) \delta(x-y)$.

With the definition of Dirac Bracket, we can compute the Dirac brackets between the fields describing the reduced Hamiltonian $H_{R}$. These are following:

$$
\begin{aligned}
{\left[A^{1}(x), \pi_{1}(y)\right]^{*} } & =\delta(x-y), \\
{[\phi(x), \phi(y)]^{*} } & =-\frac{\epsilon(x-y)}{4} . \\
{\left[\phi(x), A_{1}(y)\right]^{*} } & =0 .
\end{aligned}
$$

We see that the Dirac brackets between the fields remain canonical. From the reduced Hamiltonian (28), we calculate the following first order equations of motion with the help of Dirac brackets

$$
\begin{aligned}
& \dot{A_{1}}=\pi_{1}-\frac{1}{e^{2}\left(a-r^{2}\right)} \pi_{1}^{\prime \prime}-\frac{r}{\left(a-r^{2}\right)} A_{1}^{\prime}, \\
& \dot{\pi}_{1}=-e^{2} a \frac{\left(1+a-r^{2}\right)}{\left(a-r^{2}\right)} A_{1}-\frac{r}{\left(a-r^{2}\right)} \pi_{1}^{\prime},
\end{aligned}
$$

After some calculation Eqs. (36) and (37) reduce to the following:

$$
\begin{aligned}
& \left(\partial_{0}-\partial_{1}\right) \pi_{1}=-e^{2} a \frac{\left(1+a-r^{2}\right)}{\left(a-r^{2}\right)} A_{1}-\frac{r+1}{\left(a-r^{2}\right)} \pi_{1}^{\prime} \\
& \left(\partial_{0}+\partial_{1}\right) A_{1}=\pi_{1}-\frac{1}{e^{2}\left(a-r^{2}\right)} \pi_{1}^{\prime \prime}-\frac{r-1}{\left(a-r^{2}\right)} A_{1}^{\prime},
\end{aligned}
$$

From above two equations, we see that the $\pi_{1}$ satisfy the Klein-Gordon equation

$$
\left[\square+e^{2} \frac{a\left(1+a-r^{2}\right)}{\left(a-r^{2}\right)}\right] \pi_{1}=0,
$$

where the mass term is $m^{2}=e^{2} \frac{a\left(1+a-r^{2}\right)}{\left(a-r^{2}\right)}$. After quantization we find that the theoretical spectrum contains a massive boson with mass

$m^{2}=e^{2} \frac{a\left(1+a-r^{2}\right)}{\left(a-r^{2}\right)}$,

which is identical to the physical mass as we have obtained in Sect. 3, during the quantization of the system in Lagrangian formulation. Like the previous section no massless degrees of freedom appears here. The fermion gets confined. So the theoretical spectrum agrees in an exact manner with the theoretical spectrum obtained in Sect. 3. Imposition of chiral constraint on a model destroy the manifestly Lorentz invariance. In the next section, we will verify physical Lorentz ivariance of this model through Poincaré algebra.

\section{Poincaré algebra to show explicit Lorentz invariance}

We have already mentioned that the gauged lagrangian for chiral boson (11) does not have manifestly Lorentz covariance, but it gives Lorentz invariant theoretical spectrum. To show the physical Lorentz invariance, we need to calculate the Poincare algebra $[13,26]$. There are three elements in this algebra, the Hamiltonian $H_{R}$, the total momentum $P_{R}$ and the boost generator $M_{R}$. In the reduced phase space $P_{R}$ and $M_{R}$ reads [13]

$$
\begin{aligned}
P_{R} & =\int d x\left[\pi_{\phi} \phi^{\prime}+\pi_{1} A_{1}^{\prime}\right] . \\
M_{R} & =t\left(\pi_{\phi} \phi^{\prime}+\pi_{1} A_{1}^{\prime}\right)+\int d x\left[x H_{R}-\frac{\eta}{e^{2}\left(a-r^{2}\right)} \pi_{1}\right],
\end{aligned}
$$

where

$\eta=\pi_{1}^{\prime}+e(r+1) \phi^{\prime}+e^{2} r A_{1}$.

and the total Hamiltonian $H_{R}$ and the Hamiltonian density $\mathcal{H}_{\mathcal{R}}$ are connected by $H_{R}=\int d x \mathcal{H}_{\mathcal{R}}$. After some calcula- 
tion, it is straight forward to see that the following Poincaré algebra

$$
\begin{aligned}
{\left[P_{R}, H_{R}\right]^{*} } & =0 \\
{\left[P_{R}, M_{R}\right]^{*} } & =H_{R} \\
{\left[H_{R}, M_{R}\right]^{*} } & =\pi_{\phi} \phi^{\prime}+\left[\frac{(1+a)}{\left(a-r^{2}\right)}-\frac{\left(r^{2}+1\right)}{\left(a-r^{2}\right)}\right] \pi_{1} A_{1}^{\prime}=P_{R} .
\end{aligned}
$$

The above calculations, confirms the physical Lorentz invariance of this model.

\section{Conclusion}

Chiral constraint has been imposed into the proposed action (GVSM) and an attempt has been made to obtain a new effective action in terms of chiral boson [4-6]. Imposition of chiral constraint has spoiled the manifestly Lorentz covariance structure of the theory. However physical Lorentz invariance is found to be preserved in the physical subspace. Investigation is also carried out towards the study of its solvability. So theoretical spectrum of this model has been determined through Lagrangian and Hamitonian formulation. The spectra come in both cases exact agreement with each other.

An interesting aspect of this model is that after studying the phase space structure we have found completely different constraint structure from the constraint structure of the model discussed in [13] and drastic change in theoretical spectra is resulted. It has been found that the theoretical spectrum contains only a massive boson. Massive boson as usual can be considered as photon acquires mass. The mass of the photon is found to be identical to the mass of the massive boson as obtained in [13]. Unlike [13], massless chiral boson does not appear in the spectrum. In short, we say that the spectrum consists of a photon and the fermion gets confined.

We have done above is to prove Poincaré invariance of generalised schwinger model in terms of chiral boson by straightforwardly formulating the generators in the constrained sub space and directly verifies their obedience toward the correct algebra. We have found that despite of manifestly Lorentz covariance of this theory, the Poincaré invariance is preserved in the physical subspace. The underlying explanation is that the constraint structure of this theory is so strange that it ultimately reduced to a theory which follows Poincaré algebra correctly.

Acknowledgements I would like to thank Dr. Anisur Rahaman of Hoogly Mohsin College, for an initial discussion on the model (Generalised schwinger model).

Data Availability Statement This manuscript has no associated data or the data will not be deposited. [Authors' comment: As it is theoretical work so no data is available to be deposited.]
Open Access This article is licensed under a Creative Commons Attribution 4.0 International License, which permits use, sharing, adaptation, distribution and reproduction in any medium or format, as long as you give appropriate credit to the original author(s) and the source, provide a link to the Creative Commons licence, and indicate if changes were made. The images or other third party material in this article are included in the article's Creative Commons licence, unless indicated otherwise in a credit line to the material. If material is not included in the article's Creative Commons licence and your intended use is not permitted by statutory regulation or exceeds the permitted use, you will need to obtain permission directly from the copyright holder. To view a copy of this licence, visit http://creativecomm ons.org/licenses/by/4.0/.

Funded by SCOAP 3 .

\section{Appendix}

In Ref. [10] the Green function generating functional is multiplied by a normalization constant $\mathrm{N}$. The integration of the generating funtional over the fermionic degrees of freedom have done by authors which leads to the following lagrangian [10]:

$$
\begin{aligned}
\mathcal{L}_{\mathcal{B}}= & \frac{1}{2}\left(\dot{\phi}^{2}-\phi^{\prime 2}\right)+\frac{e}{\sqrt{\pi}}\left(A_{0} \phi^{\prime}-A_{1} \dot{\phi}\right) \\
& +\frac{e}{\sqrt{\pi}} r\left(A_{0} \dot{\phi}-A_{1} \phi^{\prime}\right)+\frac{e^{2}}{2 \pi} a\left(A_{0}^{2}-A_{1}^{2}\right) .
\end{aligned}
$$

So the Lagrange equations derived from Eq. (48) consist of $\hat{e}$ term. Authors call $\hat{e^{2}}=\frac{e^{2}}{\pi}$. Finally authors calculate the mass term after solving the equations of motion [10]. This mass term obtained in [10] contained an additional factor $\frac{1}{\pi}$. In our work the initial lagrangian is $[13,23]$

$L_{F}=\bar{\psi} \gamma^{\mu}\left[i \partial_{\mu}+e \sqrt{\pi} A_{\mu}\left(1-r \gamma_{5}\right)\right] \psi$.

The factor $\sqrt{\pi}$ is used for convenience. Conventional bosonization of (49) leads to

$$
\begin{aligned}
\mathcal{L}_{\mathcal{B}}= & \frac{1}{2}\left(\dot{\phi}^{2}-\phi^{\prime 2}\right)+e\left(A_{0} \phi^{\prime}-A_{1} \dot{\phi}\right)+\operatorname{er}\left(A_{0} \dot{\phi}-A_{1} \phi^{\prime}\right) \\
& +\frac{e^{2}}{2} a\left(A_{0}^{2}-A_{1}^{2}\right) .
\end{aligned}
$$

Calculalated equations of motion (12), (13) (14) from the lagrangian (50) do not contain $\pi$ factor. Therefore there is no additional $\frac{1}{\pi}$ factor in mass term (20) [23].

\section{References}

1. K. Harada, Phys. Rev. Lett. 64, 139 (1990)

2. W. Siegel, Nucl. Phys. B 238, 307 (1984)

3. J. Sonnenschein, Nucl. Phys . B 309752 (1988)

4. R. Jackiw, R. Rajaraman, Phys. Rev. Lett. 54, 1219 (1985)

5. S. Bcllucci, M.F.L. Golterman, D.N. Pelcher, Nucl. Phys. B 326, 307 (1989) 
6. J.M.F. Labastida, A.V. Ramallo, Vilkovisky: Phys. Lett. B 55, 224 (1975)

7. A. Rahaman, Int. J. Mod Phys A 21, 1251 (2006)

8. A. Rahaman, S. Yasmin, Ann. Phys. 383, 497 (2017)

9. A. Rahaman, S. Yasmin, S. Aziz, Int. J. Theo. Phys. 49, 2607 (2010)

10. A. Bassetto, L. Griguolo, P. Zanca, Phys. Rev. D 50, 1077 (1994)

11. A. Bassetto, L. Griguolo, Phys. Rev. D 50, 7638 (1994)

12. A. Bassetto, L. Griguolo, Nucl. Phys. B 439, 327 (1995)

13. S. Yasmin, A. Rahaman, Int. J. Theor. Phys. 55, 5172-5185 (2016)

14. S. Yasmin, A. Rahaman, Int. J. Mod. Phys. 31, 32 (2016)

15. J. Schwinger, Phys. Rev. 128, 2425 (1962)

16. J. H. Lowenstein, J. A. Swieca: Ann. Phys. (N. Y.) 68172 (1971)

17. J. Schwinger, Phys. Rev. 128, 2425 (1962)

18. C.R. Hagen, Ann. Phys. (N. Y.) 81, 67 (1973)
19. M. Chanowitz, Phys. Lett. B 171, 280 (1986)

20. I.G. Halliday, E. Rabinovici, A. Schwimmer, M. Chanowitz, Nucl. Phys. B 268, 413 (1986)

21. D. Boyanovsky, I. Schmidt, M. Golterman, Ann. Phys. (NY) $\mathbf{1 8 5}$, $111(1988)$

22. D. Sen, Phys. Lett. B 212, 191 (1988)

23. Y.-G. Miao, H.J.W. Müller-Kirsten, J.-G. Zhou, Z. Phys. C 71, 525 $-531(1996)$

24. P.A.M. Dirac, Lectures on Quantum Mechanics (Yeshiva University Press, New York, 1964)

25. K. Sundarmeyer, Constrained Dynamics (Springer, Berlin, 1982)

26. S. Ghosh, P. Mitra, Phys. Rev. D 44, 1332 (1991) 